\title{
Near-field scanning optical microscopy with monolithic silicon light emitting diode on probe tip
}

\author{
Kazunori Hoshino, ${ }^{1, a)}$ Lynn J. Rozanski, ${ }^{2}$ David A. Vanden Bout, ${ }^{2}$ and Xiaojing Zhang ${ }^{1}$ \\ ${ }^{1}$ Department of Biomedical Engineering, Microelectronics Research Center and Center for Nano \\ and Molecular Materials Science and Technology, The University of Texas at Austin, Austin, 78758 Texas \\ USA \\ ${ }^{2}$ Department of Chemistry and Biochemistry and Center for Nano and Molecular Materials Science and \\ Technology, The University of Texas at Austin, Austin, 78712 Texas USA
}

(Received 13 September 2007; accepted 8 March 2008; published online 31 March 2008)

\begin{abstract}
We describe optical and topographic imaging using a light emitting diode monolithically integrated on a silicon probe tip for near-field scanning optical microscopy (NSOM). The light emission resulted from a silicon dioxide layer buried between a phosphorus-doped $N^{+}$silicon layer and a gallium-doped $P^{+}$silicon region locally created at the tip by a focused ion beam. The tip was employed in a standard NSOM excitation setup. The probe successfully measured optical as well as topographic images of a chromium test pattern with imaging resolutions of 400 and $50 \mathrm{~nm}$, respectively. The directional resolution dependence of the acquired images directly corresponds to the shape, size, and polarity of the light source on the probe tip. To our knowledge, this report is the first successful near-field imaging result directly measured by such tip-embedded light sources.

(C) 2008 American Institute of Physics. [DOI: 10.1063/1.2904698]
\end{abstract}

Near-field scanning optical microscopy (NSOM) measures optical properties on a subwavelength scale with simultaneous topography imaging ${ }^{1}$ and is a powerful tool for exploring nanoscale structures such as inorganic nanoparticles, ${ }^{2}$ organic thin films, ${ }^{3,4}$ and interfaces of biomaterials. ${ }^{5}$ A pulled optical fiber with an external laser light source was typically used in NSOM. ${ }^{1}$ Direct integration of light source, in particular, monolithic integration of an electrically pumped light source on scanning probe is highly desirable to further extend the applications of NSOM into otherwise difficult-toaccess environments, such as compact pressure or temperature-controlled chambers. ${ }^{6}$ Silicon-monolithic light sources, in combination with the integrated circuit technology, will also lead to future data storage devices employing near-field optics. ${ }^{7}$ Here, we describe an imaging result of NSOM taken with a nanometer-sized light emitting diode (LED) monolithically integrated on a silicon probe tip. To our knowledge, this report is the first successful near-field imaging result directly measured by such tip-embedded light sources. Another unique feature of our method is that the imaging characteristics are directly related to the size of the apertureless built-in light source. As a comparison, the optical resolution of most NSOM systems is defined by the diameter of an aperture created through a metal coated glass fiber optic ${ }^{1}$ or a silicon-based microprobe. ${ }^{7,8}$ This structure causes large energy dissipation at the probe tip. There have been previous efforts to create scanning probes integrated with light sources, but some are large and have not fully functioned as a scanning light source in a working NSOM setup, ${ }^{9}$ and others are not monolithically integrated and still rely on an aperture created through a metal coating to define the light source size. ${ }^{10,11}$

Ion doping with focused ion beams (FIB) allows for the creation of nanometer-sized $P-N$ diodes. ${ }^{12,13}$ LEDs employing InAs quantum dots in a FIB-created $P N$ junction have

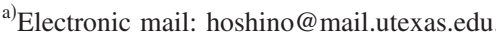

also been reported. ${ }^{14,15}$ In this paper, we used a FIB to create a nanometer-scale LED on a microfabricated silicon scanning probe tip. The FIB plays two important roles. First, ion milling was used to reshape the probe tip and to create the electrodes at the very tip. Second, FIB-induced redeposition and doping were used to create a nanometer sized $P^{+}$region for the LED. Figure 1 shows the experimental NSOM setup (Veeco Aurora system). The probe is attached to a quartz tuning fork ${ }^{16}$ and oscillated by an external piezoactuator at a resonant frequency of $95-100 \mathrm{kHz}$. As the probe tip approaches the sample surface, a drag force acting on the tip damps the oscillation, providing feedback signal for use in tip-sample distance control. For optical excitation, the nearfield light source is a nanoscale LED created on the probe tip. A microscope objective is located below the sample to collect the light which is subsequently imaged onto a single photon counting detector. While the probe tip scans the sample surface to acquire topography, an optical image was simultaneously acquired from the intensity of transmitted light.

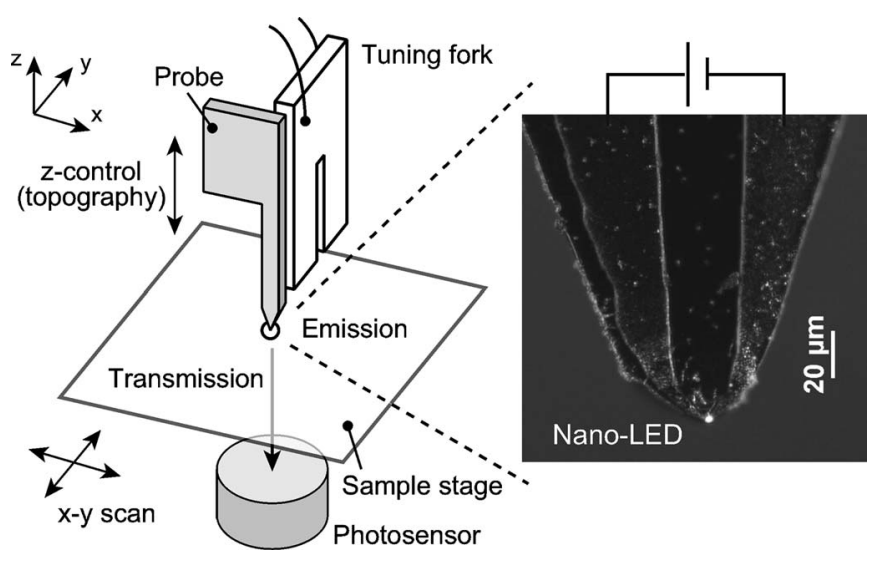

FIG. 1. NSOM setup. A tuning fork was used to maintain tip-sample distance roughly at 5-10 $\mathrm{nm}$. Optical intensity was measured in transmission mode. 


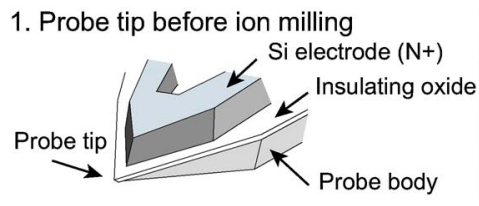

2. Ion milling of the electrode

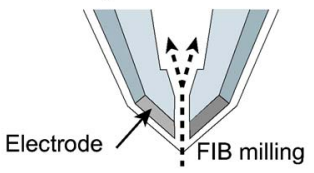

3. Redeposition of Ga-doped Silicon
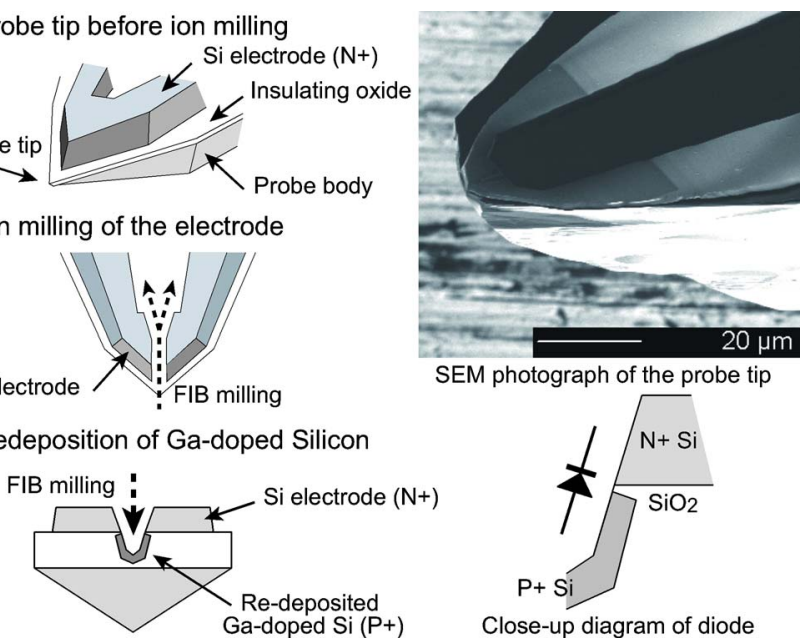

SEM photograph of the probe tip

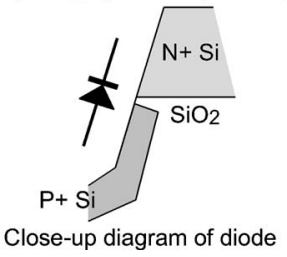

FIG. 2. (Color online) LED fabricated on the silicon probe. A silicon dioxide layer created between layers of phosphorus doped $N^{+}$silicon and gallium doped $P^{+}$silicon worked as a light emitter.

Figure 2 shows the composition of the silicon microprobe. The detailed fabrication procedure was reported elsewhere. ${ }^{17}$ The probe was made of a silicon-on-insulator wafer with a $2-\mu \mathrm{m}$-thick device layer, which was doped by phosphorous oxychloride for $50 \mathrm{~min}$ at $900{ }^{\circ} \mathrm{C}$. First, the electrode pattern as well as the probe body were created through wet etching. The probe tip was then trimmed with a FIB so that the tip pair of the electrodes converges to the very tip of the probe body. Patterning and positioning precision is typically $100-200 \mathrm{~nm}$. Then, the FIB was again used to form a 150-nm-wide gap on the electrode tip. This milling process induces redeposition ${ }^{18}$ of gallium doped $p$-type silicon, which eventually creates a thin silicon dioxide layer as the light emitter shown in Fig. 2. According to the previous studies on $\mathrm{Si} / \mathrm{SiO}_{2}$ LEDs, ${ }^{19,20}$ the thickness of the buried silicon dioxide is estimated to be less than $100 \mathrm{~nm}$.

Figure 3 shows the $\mathrm{Si} / \mathrm{SiO}_{2}$ LED's basic characteristics including $I-V$ (current-voltage), $L-V$ (light intensity-voltage), and $L-I$ (light intensity-current) curves. Clear linearity in the $L-I$ curve indicates the successful electroluminescence emitted from the light source. A peak emission wavelength at $575 \mathrm{~nm}$ was observed to be independent of the applied current, with a broad spectrum of the visible wavelengths similar to the characteristics reported for the $\mathrm{Si} / \mathrm{SiO}_{2}$ interface LED. ${ }^{19,20}$ Several reports have been made to tailor emission
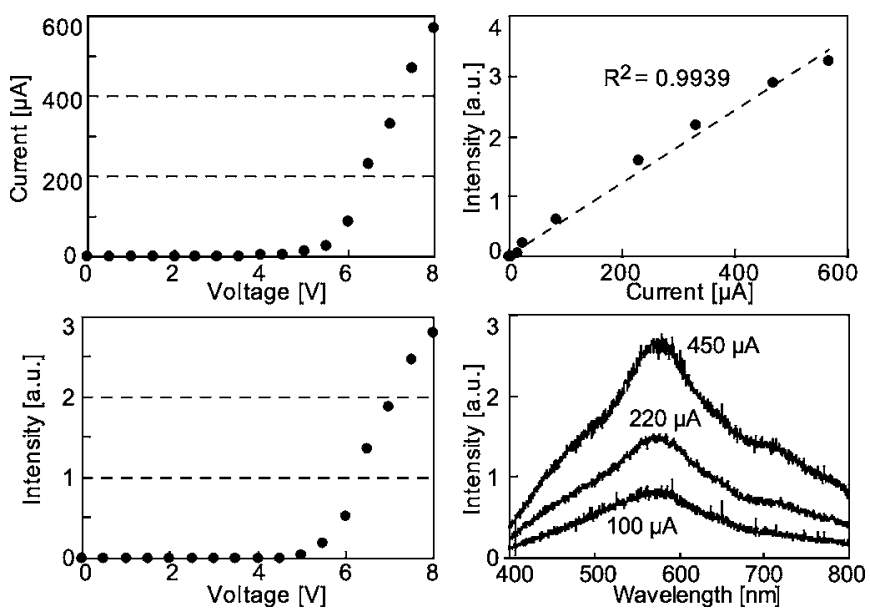

FIG. 3. Characteristics of the nanoscale LED.

Downloaded 02 Apr 2008 to 128.83.154.109. Redistr
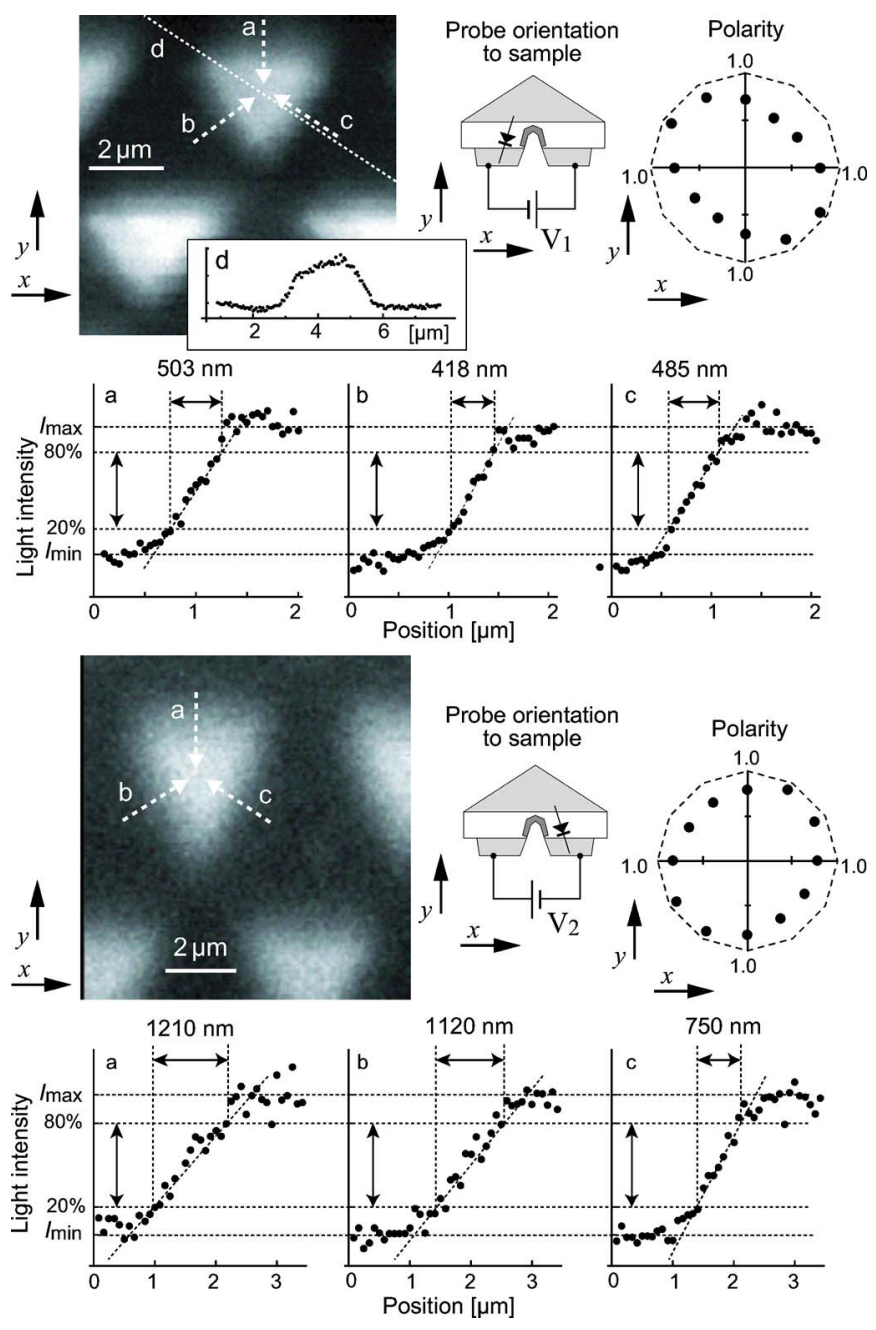

FIG. 4. (Color online) Optical images taken with two different probes with different voltage polarities. The light intensity slopes showed angular dependence of resolution which can be correlated to the polarity of the light source.

wavelengths of $\mathrm{Si} / \mathrm{SiO}_{2}$ LEDs. Semiconductor nanoparticles introduced between a FIB-cut gap modified the emission wavelength. ${ }^{21}$ Violet electroluminescence from a germanium-doped $\mathrm{Si} / \mathrm{SiO}_{2} \mathrm{LED}$ was utilized for fluorescent measurement. ${ }^{22}$ Both methods are well compatible with our probe design and fabrication.

Figure 4 shows imaging results of electroluminescence through a chromium test pattern made by e-beam lithography on a glass substrate. Illustrations of the probe section are shown to indicate the probe orientation to the sample. Two different probes driven with opposite voltage polarity to one another (V1, V2) were used. An analyzer polarizer placed in front of the detector was used to measure the polarization of light emission from each probe. It was determined that both probes had symmetrically polarized light emission with respect to the $y$ axis. The polarization aligned in the different directions for the two probes is likely due to both the opposite polarity of the applied voltages and the geometry of the LED in the probe tip (see Fig. 2 for the probe geometry). Intensity profiles along the orthogonal directions $(a, b$, and $c$ ) to each side of the triangular pattern were compared to assess the directional dependence of the resolution. The slopes of intensity variation along $a, b$, and $c$ were calculated from fit lines of the points measured between $20 \%$ and $80 \%$ of the maximum intensity. In both cases, the best resolution is o AIP license or copyright; see http://apl.aip.org/apl/copyright.jsp 


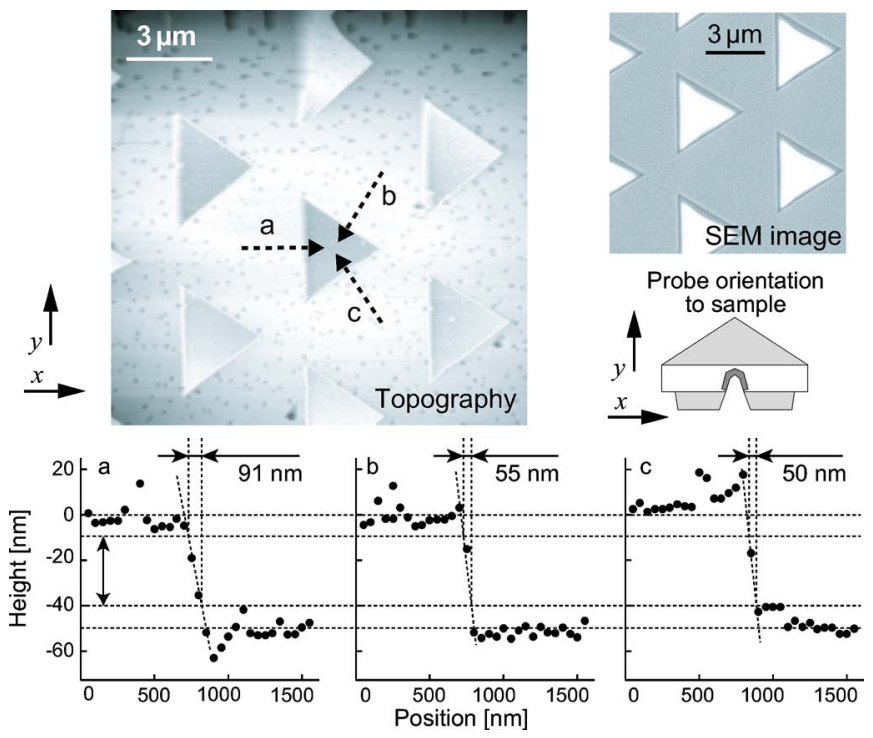

FIG. 5. (Color online) Topographic image taken by the probe. SEM image of the chromium pattern is on the upper right panel. Topographic resolution was low in $y$ direction.

obtained for a line perpendicular to the polarity of the light sources. The profile along line $d$ showed a trapezoidal shape with the flat top side. This indicates that the light source size is small enough compared to that of the triangle pattern and the measured profile mainly resulted from optical signal which was not coupled with the topography. A topographic image was also taken for the same chromium pattern. The thickness of the chromium was $30 \mathrm{~nm}$. Profiles along three lines were compared in an identical manner to the optical images. In cases $b$ and $c$, only one point was found in $20 \%-$ $80 \%$ of the maximum height, and a neighboring point on each side was taken into account. Directional dependence was also observed, which suggests that the nanometric shape of the probe tip is wider in the $x$ direction, similar to the section of the probe shown in Fig. 5.

The capability of simultaneous topographical and optical imaging is one of the strong advantages of NSOM. Figure 6 shows the comparison of topography and the optical intensity of the sample taken at the same time. Image shift between the two was measured as $\Delta x=180 \mathrm{~nm}$ and $\Delta y$ $=700 \mathrm{~nm}$. The shift may be caused by the variation of the

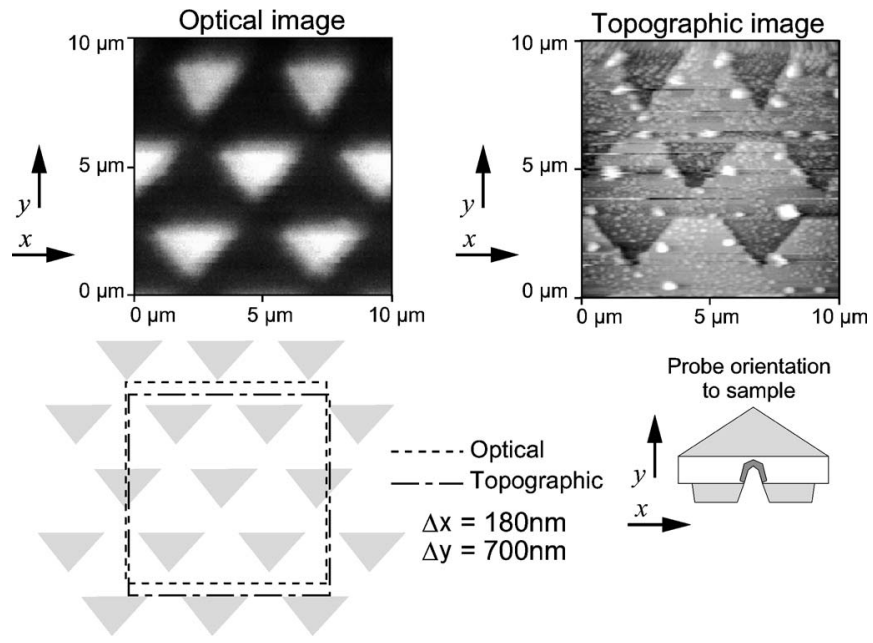

FIG. 6. Optical and topographic images of a chromium target taken at the same scan. Larger image shift was observed in $y$ direction, which corresponds to the direction of FIB milling.

Downloaded 02 Apr 2008 to 128.83.154.109. Redistribution subject to AIP license or copyright; see http://apl.aip.org/apl/copyright.jsp
nano-LED and probe-tip separation, waveguiding effect along the $\mathrm{Si} / \mathrm{SiO}_{2}$ layers, or both. Because FIB milling procedure provides less control along the depth comparing to the lateral directions, distance variation between the physical probe tip and the light source is larger along the depth, i.e., the $y$ direction of the sample. It is also possible that the insulating oxide layer, which has the thickness of $1.1 \mu \mathrm{m}$, works as a waveguide to widen the light source size. Since the obtained optical resolution and the image shift are considerably smaller than the oxide thickness, the wave-guiding effect is likely to be less significant. The result suggests that the image shift should mainly depend on the location of the light source on the tip, and therefore can be well controlled by the further exploration of multidimensional freedom $(x, y$, and $z$, rotation and tilt) of the mechanical stage employed in the FIB apparatus.

In summary, we have made an NSOM light source monolithically integrated at a silicon probe tip. This probe has been used to collect simultaneous optical and topographic images of a chromium test pattern with resolutions of 400 and $50 \mathrm{~nm}$, respectively. The images reveal a directional resolution dependence that corresponds to the shape, size, and polarity of the light source on the probe tip. As the resolution is directly related to the light source size, it therefore can be further improved by refining fabrication procedures.

We thank the Wallace H. Coulter Foundation, National Science Foundation (ECS-0609413 and ECS-26112892), and UT Research Grant.

${ }^{1}$ B. Hecht, J. Chem. Phys. 112, 7761 (2000).

${ }^{2}$ S. R. Emory and S. Nie, Proc. Natl. Acad. Sci. U.S.A. 93, 6264 (1996). ${ }^{3}$ P. F. Barbara, D. M. Adams, and D. B. O'Connor, Annu. Rev. Mater. Sci. 29, 433 (1999).

${ }^{4}$ J. Teetsov and D. A. Vanden Bout, J. Phys. Chem. B 104, 9378 (2000).

${ }^{5}$ M. F. Garcia-Parajo, J. A. Veerman, S. J. T. van Noort, B. G. de Grooth, J. Greve, and N. F. van Hulst, Bioimaging 6, 43 (1998).

${ }^{6}$ G. Behme, A. Richter, M. Süptitz, and Ch. Lienau, Rev. Sci. Instrum. 68, 3458 (1997).

${ }^{7}$ M. Sasaki, K. Tanaka, and K. Hane, Jpn. J. Appl. Phys., Part 1 39, 7150 (2000).

${ }^{8}$ P. N. Minh, T. Ono, and M. Esashi, Appl. Phys. Lett. 75, 4076 (1999).

${ }^{9}$ K. H. An, B. O'Connor, K. P. Pipe, Y. Zhao, and M. Shtein, Appl. Phys. Lett. 89, 111117 (2006).

${ }^{10}$ S. Heisig, O. Rudow, and E. Oesterschulze, Appl. Phys. Lett. 77, 1071 (2000).

${ }^{11}$ S. Khalfallah, C. Gorecki, J. Podlecki, M. Nishioka, H. Kawakatsu, and Y. Arakawa, Appl. Phys. A: Mater. Sci. Process. A71, 223 (2000).

${ }^{12}$ A. J. Steckl, H. C. Mogul, and S. Mogren, J. Vac. Sci. Technol. B 9, 2718 (1991).

${ }^{13}$ H. C. Mogul, A. J. Steckl, and E. Ganin, IEEE Trans. Electron Devices 40, 1823 (1993).

${ }^{14}$ M. Vitzethum, R. Schmidt, P. Kiesel, P. Schafmeister, D. Reuter, A. D. Wieck, and G. H. Döler, Physica E (Amsterdam) 13, 143 (2002).

${ }^{15}$ R. Schmidt, U. Scholz, M. Vitzethum, R. Fix, C. Metzner, P. Kailuweit, D. Reuter, A. Wieck, M. C. Hübner, and S. Stufler, Appl. Phys. Lett. 88, 121115 (2006).

${ }^{16}$ K. Karrai and R. D. Grober, Appl. Phys. Lett. 66, 1842 (1995).

${ }^{17}$ K. Hoshino, L. J. Rozanski, D. A. Vanden Bout, and X. J. Zhang, J. Microelectromech. Syst. 17, 4 (2008).

${ }^{18}$ W. Boxleitner and G. Hobler, Nucl. Instrum. Methods Phys. Res. B 180, 125 (2001)

${ }^{19}$ L. Heikkil, T. Kuusela, and H. P. Hedman, J. Appl. Phys. 89, 2179 (2001).

${ }^{20}$ T. Matsuda, M. Nishio, T. Ohzone, and H. Hori, Solid-State Electron. 41, 887 (1997).

${ }^{21}$ K. Hoshino, K. Yamada, K. Matsumoto, and I. Shimoyama, J. Micromech. Microeng. 16, 1285 (2006).

${ }^{22}$ L. Rebohle, T. Gebel, R. A. Yankov, T. Trautmann, W. Skorupa, J. Sun, G. Gauglitz, and R. Frank, Opt. Mater. (Amsterdam, Neth.) 27, 1055 (2005). 